ISSN: 2602-8506

Vol. 3, N4., p.116-132, octubre - diciembre, 2019

\title{
Big Data en la gestión del talento humano
}

\section{Big Data in human talent management}

\section{Washington Edy Santillán Marroquín. ${ }^{1}$, Hernán Eduardo Paredes García. ${ }^{2}$, César Armando Bermeo Castillo. ${ }^{3}$, Héctor Patricio Mera Manotoa. ${ }^{4}$ \\ DOI: https://doi.org/10.33262/visionariodigital.v3i4.984}

\section{Abstract}

The new times, the technological era also called "the information age" in which, society, customers and companies must adapt to a new changing world generating and trying to process more and more data, which in many cases are impossible Sometimes they are even unimaginable because of the proportion of them. In order to acquire and analyze so much information, the term Big Data arises, a term that has represented confusion because it has no limits in its scope.

In this article we try to know this term and why Big Data arises, where it comes from and why for many new entrepreneurs and administrators suggests a change of direction in the world of Tics.

In the same way we will focus on the use of Big Data in Human Talent Management, its way of being measured and the pros and cons of having this information at present.

Keywords: Information, administration, society, clients, companies

\section{Resumen}

Los nuevos tiempos, la era tecnológica también llamada "la era de la información” en la cual, la sociedad, los clientes y las empresas deben adaptarse a un mundo nuevo cambiante generando e intentando procesar más y más datos, que en muchos casos son imposibles de manejar a veces hasta son inimaginables por la proporción de los mismos. Para lograr adquirir

1 Coordinador académico Instituto Ohiggins, Quito, Ecuador, coordinacion.academica@instituto-ohiggins.com

2 Rector Instituto Superior Tecnológico Bernardo Ohiggins, Quito, rectorado@institutoohiggins.com

3 Docente de Administración Instituto Ohiggins. Quito, cesararmando.bermeo@ hotmail.com

4 Docente de Administración Instituto Ohiggins. Quito, patomera@ hotmail.com 
y analizar tanta información surge el término Big Data, término que ha representado una confusión por no tener límites en su alcance.

En este artículo tratamos de conocer este término y porque surge el Big Data, de donde procede y por que para muchos nuevos empresarios y administradores sugiere un cambio de rumbo en el mundo de las Tics.

De igual manera nos enfocaremos en la utilización del Big Data en la Gestión del talento humano su forma de ser medido y los pro y contras de contar con esta información en la actualidad.

Palabras claves: Información, administración, sociedad, clientes, empresas

\section{Introducción}

En estos tiempos donde la tecnología ha rebasado lo inimaginable nuestra vida está siendo observada desde el mismo momento que ponemos un pie fuera de nuestra casa, estamos sin darnos cuenta generando datos.

Prendemos nuestro teléfono inteligente (Smartphone) que dentro de sus aplicaciones tiene Google maps, GPS, correo electrónico, redes sociales, cuando usas una tarjeta de crédito, o realizas compras en línea estas generando datos, produciendo información digital. Esto ha llevado a la popularización del término Big-data hace muy pocos años sobre todo por la utilización de toda esta información por parte de las grandes empresas de tecnología para facilitar sus bases de datos y proponer mejor servicio al cliente apuntando a los mejores historiales de crédito y hacer las inversiones seguras. Esto demando la recolección de toda esa información clasificarla y tenerla a disposición, sin embargo, al mismo tiempo que resulta beneficioso podría ser catalogado de invasivo.

Así nacen muchas preguntas ¿Es la proliferación de datos la prueba de que el mundo es cada vez más intrusivo? ¿Podemos estar seguros de que hay un peso y un valor económico detrás de toda esta información masiva? ¿Debe existir una ley que regule esto? Etc. Muchas preguntas que debemos resolver.

En el campo empresarial es un hecho que el Big Data debe estar presente, pero en la gestión de talento humano ¿Qué nos aporta? ¿Para qué nos servirá? 
Vol. 3, N4., p.116-132, octubre - diciembre, 2019

En la gestión de talento humano esta gran cantidad de datos nos aportan en que puedan ser tratados y analizados para la extracción de información relevante para generar informes y predicciones.

En el año 1998 se afirmó que el volumen de datos estaba creciendo a un ritmo nunca visto anteriormente. A este ritmo sería imposible de manejar por parte de las infraestructuras físicas y humanas empleadas en ese año. Por este motivo, las empresas se vieron obligadas a adaptarse a estos nuevos cambios.

El Big Data aporta una serie de utilidades para los profesionales de los recursos humanos. Gracias a una gestión adecuada de los datos, es posible recopilar toda la información que manejan de forma correcta y organizada. Pero, ¿qué ventajas ofrece?

\section{Marco Teórico}

¿Qué es Big Data?

Big Data, del cual existen innumerables definiciones, entre ellas se tienen: Según el término aplica a la información que no puede ser procesada o analizada mediante procesos tradicionales. Grande, L. S. V. (2004)

Para thinkupapp.com, (2012), Big Data son "cantidades masivas de datos que se acumulan con el tiempo que son difíciles de analizar y manejar utilizando herramientas comunes de gestión de bases de datos", y para E. Dans (2011) Big Data se refiere "al tratamiento y análisis de enormes repositorios de datos, tan desproporcionadamente grandes que resulta imposible tratarlos con las herramientas de bases de datos y analíticas convencionales".

En B. Hopkins (2011) Forrester define Big Data como las técnicas y tecnologías que hacen que sea económico hacer frente a los datos a una escala extrema. Big Data trata de tres cosas:

1) Las técnicas y la tecnología, lo que significa que la empresa tenga personal, el cual tenga gran representación y análisis de datos para tener un valor agregado con información que no ha sido manejada.

2) Escala extrema de datos que supera a la tecnología actual debido a su volumen, velocidad y variedad. 
Vol. 3, N4., p.116-132, octubre - diciembre, 2019

3) El valor económico, haciendo que las soluciones sean asequibles y ayuden a la inversión de los negocios".(Vega, 2015)

Según Zdnet.com, (2010 Big Data) "se refiere a las herramientas, los procesos y procedimientos que permitan a una organización crear, manipular y gestionar conjuntos de datos muy grandes y las instalaciones de almacenamiento". Gartner (2011) define el Big Data como "un gran volumen, velocidad o variedad de información que demanda formas costeables e innovadoras de procesamiento de información que permitan ideas extendidas, toma de decisiones y automatización del proceso". (Vega, 2015)

La sociedad crea datos y más datos y cada vez existen más dispositivos (Discos duro, pendrive de diferentes capacidades) y más eficientes para almacenarlos. Los datos son vistos un capital en sí mismos para la organización ya sea pública o privada es una gran inversión tener acceso a ellos y la empresa obtiene poder con la información almacenada. Volviéndose indispensable el que esté al alcance de todos.

\section{¿Cómo Afecta a la Sociedad el Big Data?}

Cómo afecta el Big-data a la sociedad en general No sólo son importantes los datos y el conocimiento que nos aportan los mismos (Monleón, 2010), sino que están cambiando la economía mundial.

En América Latina la información en la Red al igual que el resto del mundo está formado por datos estructurados como las tradicionales bases de datos que poseen las empresas, por ejemplo, una empresa de servicios pude tener el histórico sobre el consumo de energía de un usuario, y también existe la información con datos desestructurados, que en cantidad superan tres veces a los primeros. Buena parte de la información desestructurada que representa el $80 \%$ del total-proviene de las herramientas sociales.

Para tener una idea de lo que pasa en la Red en sólo un minuto: se generan 98.000 tweets, se bajan 23.148 aplicaciones, se juegan 208.333 minutos de Angry Birds, 277.000 personas se loguean a Facebook y se ven 1,3 millones de videos en YouTube, entre otras decenas de métricas (mediciones) que alimentan sin cesar la nube virtual y le enseñan sobre nuestra conducta online a quien lo requiera. "Todas las industrias guardan y necesitan acceder a la información de maneras muy distintas - describe Marcelo Fandiño, CEO de EMC Argentina, empresa de almacenamiento y cómputo en la nube. Algunos datos necesitan ser analizados en 
tiempo real, otros estar almacenados por años con consultas muy puntuales, en Ecuador esto ya se utiliza por ejemplo en el Registro Civil, en el Consejo de la Judicatura etc. Ahora tenemos una memoria gigante y lo que hay que hacer es darle toda la inteligencia. El usuario se está volcando cada vez más a la Red para tener su información y pronto tendrá su máquina principal completamente alojada en la nube. Para esto tendremos que darle el máximo y más creativo análisis a cada documento y dato alojados allí." (Mahiques, 2012)

No es casualidad que, en el Foro Económico Mundial celebrado en junio en Suiza, el concepto de Big Data haya sido protagonista. Un informe desarrollado durante el encuentro, llamado Big Data, Big Impact, declara a la masa de información gigante como una nueva clase de activo económico, como el dinero o el oro. Sebastián Bellagamba, director para Latinoamérica y el Caribe de la Internet Society, organismo mundial que vela por el buen desarrollo y crecimiento de Internet, reflexiona sobre el fenómeno:

"No es la primera vez que se juntan datos nuestros, uno ha estado dando información buena parte de su vida, sólo que internet lo hace más evidente. La Red va coleccionando datos de nuestro perfil (sexo, edad, gustos, hábitos, procedencia); eso sirve para darnos mejores resultados en las búsquedas y que pueden servir para tomar decisiones o generar políticas públicas que impacten de manera positiva en la sociedad, que no es lo mismo que juntar y distribuir datos personales como número de DNI, dirección o nombre. Que la información de la Red crezca es deseable siempre que se respeten las libertades individuales y el usuario pueda elegir qué datos le da al universo virtual", advierte Bellagamba. (Rua, Zona de Promesas, s.f.)

Desde IBM explican el universo de Big Data a partir de tres v:

Volumen, Variedad y Velocidad.

"Tenemos un gran desafío por delante, dado que se espera que para el año 2020 haya 35 zettabytes de información. Con IBM Insights trabajamos con plataformas de código abierto, le agregamos seguridad y el análisis es cada vez más intuitivo; intenta encontrar patrones y utiliza herramientas para conectar de esta información que proviene de lugares diferentes. El análisis tradicional de Business Intelligence (inteligencia de datos) ya no es suficiente. Si una telefónica sufre una caída de servicio 
y los usuarios empiezan a reclamar en las redes, debe poder acceder a esa información de inmediato para actuar cuanto antes" (Rua, La Nación, 2012)

Hemos visto solo la descripción de este fenómeno del que toda persona que navega en la web es parte así sea que su tiempo dentro de la misma varíe, entonces nos planteamos la interrogante: ¿Debería el Big Data interesarles a las personas? o ¿Afecta que la información de su vida esté expuesta en la nube?

Y la preocupación no va en sentido de que los datos estén allí sino en la cantidad de datos nuevos que recibe la web, creíbles y no creíbles. En la actualidad, las empresas y los gobiernos trabajan con miles de sensores digitales que arrojan información de todo tipo a la Red. En equipos industriales como: Automóviles, electrodomésticos, en las calles, estos sensores pueden medir y comunicar la localización, el movimiento, la vibración, la temperatura, la humedad y hasta cambios químicos en el aire. Esto, y la actividad en la Red en general, permite la toma de decisiones: qué construir y dónde hacerlo, prevenir un desastre natural, evitar un embotellamiento, detectar posibles derrumbes, medir la cantidad de tiempo que permaneces dentro de la web si serás productivo o no, tus intereses etc.

"Es un fenómeno creciente, que antes existía, pero se daba en ambientes controlados con datos estructurados; ahora los datos provienen de todos lados. Google no existiría sin toda esa Big Data y es nuestra misión organizarla y entregarla a los usuarios de una manera que sea relevante para su vida". (Rua, La Nación , 2012)

Cualquier usuario puede también consultar y usar la inteligencia que se desprende de la Web. En Buenos Aires y muchas ciudades de la Argentina y el resto del mundo, cualquier usuario puede ir a Google Maps, introducir una dirección, elegir la vista de satélite y ver en tiempo real la congestión de tráfico de la zona que desea visitar, con información que los mismos usuarios envían a la Red por sólo usar su teléfono Android. También Google ha descubierto que ciertos términos de búsqueda sirven como buenos indicadores de la actividad de la gripe y desarrolló Flue Trends, donde cualquiera puede ingresar y ver la evolución de la gripe a través de datos globales de las búsquedas de los internautas en Google. Así, se pueden hacer cálculos aproximados de la actividad de esta enfermedad en determinadas regiones, lo que podría resultar de utilidad para tomar acciones de prevención (Larrosa, s.f.) 
También se puede usar la información para hacer más eficiente el consumo energético. "A través de la estrategia de Smart Meters de IBM, se analizó el consumo de electricidad de un barrio a través de sensores que enviaban datos de consumo. En base a esa información, la compañía fue capaz de determinar los hábitos de los vecinos en cada momento del día, ver cómo variaba la demanda y hasta cambiar algunos de esos hábitos con estrategias de premios y bonificaciones a sus clientes" (Larrosa, s.f.)

Por otro lado, de Big Data la ventaja del ciudadano común es que se tiene cada vez más acceso a ver datos de gobierno que antes sólo eran analizados al interior de las administraciones públicas. En 2009, la ciudad de Washington fue la pionera en este sentido y abrió las puertas a toda su información con Data.gov. En marzo de este mismo año el GCBA implementó Buenos Aires Data, el primer repositorio público de datos abiertos del país basado en CKAN plataforma utilizada por otros gobiernos nacionales y municipales alrededor del mundo. En Ecuador en el año 2009 de igual manera se aprobó el reglamento de la LOTAIP Ley Orgánica de Transparencia y Acceso a la Información Pública que mandaba a que las empresas del estado coloquen su información en la web desde los contratos de personal, organigramas, sueldos hasta las partidas presupuestarias para compras e inversiones.

De igual manera los GADS están en la obligación de colocar toda esta información y el ciudadano tiene el acceso a la misma. A la luz de todo esto, no existe en la actualidad empresa que despliegue su estrategia de negocio sin contemplar una mirada sobre Big Data. Ya sean de hardware, software o servicios, todas apuntan sus desarrollos e inversiones a herramientas y aplicaciones que puedan desarmar el ovillo más grande del mundo (Rua, La Nación, 2012). Así EMC, HP, IBM, Netapp, Intel, SAP, entre tantas, lanzan herramientas para llegar a esos datos de manera más rápida y eficiente cosas que se buscaban en semanas hoy se lo hace en un día gracias a estas herramientas. Por otra parte, las empresas gigantes de Internet como Facebook, Google y Amazon poseen herramientas propias para analizar la información, y las consultoras de análisis Web trabajan a destajo en aumentar el cruzamiento de estos datos estructurados y no estructurados en busca de un mejor aprovechamiento del universo virtual. No seremos sólo testigos de este fenómeno, sino protagonistas de la era del nuevo oro, el oro de la información en la nube (Rua, Zona de Promesas, s.f.). 
Vol. 3, N4., p.116-132, octubre - diciembre, 2019

\section{Utilidad de Big Data en Talento Humano}

Pero que utilidad genera el Big Data a la gestión de talento humano pues bien analizaremos el sin fin de oportunidades y utilidades que esto representa ya que gracias a una gestión adecuada de los datos es posible recopilar toda la información que manejan de forma correcta y organizada (Ranstad, 2016).

De acuerdo a datos de consultoras como Deloitte y PWC, 9 de cada 10 empresas crean informes para valorar correctamente el análisis de talento humano, pero uno de cada tres directores de Talento Humano tiene dificultades para evaluar qué datos son realmente útiles. Hay altas expectativas, puesto que la tendencia es la de utilizar todos los datos existentes (estructurados y no estructurados). El resultado es que dos de cada tres empresas están invirtiendo en soluciones de análisis de datos, y el $31 \%$ de las organizaciones están contratando especialistas para sus equipos de análisis (Mihala, 2019).

Permite obtener información valiosa sobre la persona que se ha seleccionado o se va a seleccionar para la empresa. La ayuda que en este momento aportan las herramientas sociales (redes), que permiten gestionar el talento de las personas. Tomando en cuenta que podemos ver su conducta en la nueva sociedad cibernética si este individuo es apto para trabajar en nuestras empresas, de allí se extrae los currículos que recibe como de la información a la que tiene acceso por su cuenta, se puede llevar a cabo un proceso de selección de personal más analítico y estratégico. También permite elegir con más precisión el tipo de perfil que se necesita (Ranstad, 2016).

Hoy todo es medible desde el ordenador mediante encuestas, evaluaciones o entrevistas se puede conocer si el equipo está, o no, motivado; o el nivel de satisfacción de los empleados con la empresa. De igual forma, es posible identificar problemas puntuales de una compañía y llevar a cabo estrategias específicas para solucionarlos (Ranstad, 2016).

Se puede ejecutar estrategias de emprendimiento planes de carrera de acuerdo a las motivaciones que tienen los empleados y que las expresan en sus redes, podemos indagar sobre sus preocupaciones y las carencias que pueda tener en su puesto de trabajo así mismo averiguar si las oportunidades de desarrollo que ofrecen algunas empresas están siendo aprovechadas, o no, por sus integrantes como por ejemplo plataformas de E- learning, 
Vol. 3, N4., p.116-132, octubre - diciembre, 2019

visitadas en horarios de trabajo, páginas de motivación o accesos a nuevas oportunidades laborales.

La cambiante fuerza laboral presiona a que las cabezas de las áreas de Talento humano a busquen ideas que permitan predecir tendencias y obtener resultados positivos en el reclutamiento y selección de nuevo personal.

Estas cifras muestran que el Big Data -el análisis sistemático de grandes volúmenes de datos para la toma de decisiones, está cobrando una gran importancia en las empresas y prometen revolucionar las estrategias de los departamentos de talento humano. Pero ¿cómo iniciar un proyecto Big Data con éxito en el área de talento humano? Estos son los pasos que Oracle ha identificado y que deben dar las organizaciones (Mihala, 2019).

\section{Identificar: sea claro y limpio}

Hay que tomarse el tiempo necesario para identificar qué tipo de datos se necesita realmente para tener éxito en su estrategia de talento humano. En la actualidad estos departamentos tienen a menudo islas de datos sin conectividad externa es decir muy limitada, lo que restringe la capacidad del usuario para utilizar esos datos en diferentes dispositivos y herramientas. Un proyecto de Big Data es la oportunidad para identificar dónde están las islas de datos y definir una estrategia para integrarlas, limpiarlas y ordenarlas (Calle, 2018).

La importancia de los datos dependerá de los objetivos buscados es decir puede ser relativa. Si la empresa quiere mejorar la movilidad interna, hay que asegurarse de que puede obtener los datos pertinentes para que cada departamento pueda contar con la información necesaria de potenciales candidatos internos (Mihala, 2019).

Hay que crear una interfaz para todos para evitar prácticas de "copiar y pegar" y asegurarse de que los datos se recojan de forma automática y bien organizada en una interfaz. Cuanto menos se tenga que trabajar ocupando tiempo en la búsqueda de datos de forma manual para integrarlos en los sistemas de talento humano, más correctos y útiles serán. Así, es necesario escoger una única interfaz para recopilar los datos que sea de fácil acceso para todo el equipo.

Se debe apostar por la simplicidad y la eficiencia escogido una vez el tipo de datos que necesita la empresa, en la forma y donde se recogen, es necesario decidir qué tipo de análisis necesita. Para ello, es importante recordar el valor de la simplicidad, comenzando con el 
análisis de correlaciones simples que permitan comprender los mecanismos y los tipos de resultados que se podrían obtener.

Apostamos por la simplicidad, es fácil entender el poder de Big Data al obtener magníficos análisis predictivos, y esto es un activo fundamental para los departamentos de Talento humano. El uso de Big Data mejorará la estrategia y la contribución del talento humano al negocio. Pero hay que ir paso por paso, en caso contrario, los Big Data serán otro problema que hay que gestionar.

Ahora con esos datos disponibles, puede trabajar de la siguiente manera:

- Tener visibilidad más precisa de predecir y planificar el desempeño organizacional

- Minimizar el costo, el tiempo y el riesgo de asumir nuevas iniciativas de recursos humanos

- Tener una sola fuente de la tecnología permita comprender, desarrollar y mantener una fuerza de trabajo productiva

- Darle informes al CEO exactamente lo que la fuerza de trabajo está contribuyendo a la empresa mediante la cuantificación del ROI

- Tener una visión multifuncional de múltiples fuentes de datos todo en un solo tablero de mando (Grupo Castilla, 2016).

La mejor alternativa es apoyar el desempeño del negocio mediante la comprensión de la real fuerza de trabajo global y gestionando estrategias de talento humano específicos, no sólo para predecir el rendimiento y el desgaste, sino también para determinar las acciones correctivas, detectar anomalías para una ganancia inmediata en riesgo inherente (Mihala, 2019).

Crear ventajas asociadas a la fuerza de trabajo, es decir, obteniendo puntos de vista del rendimiento de los empleados, el potencial, la alineación de las habilidades con los objetivos, y las perspectivas de sucesión en puestos clave.

A través del análisis de Big Data, los responsables de talento humano estarán en condiciones de crear y visualizar correlaciones profundas entre los datos, mejorando entonces la eficiencia del negocio.

\section{La lluvia de oportunidades}

Muchas empresas en nuestro país dan el soporte necesario para el manejo de los datos grandes, tal es el caso de ORACLE, 


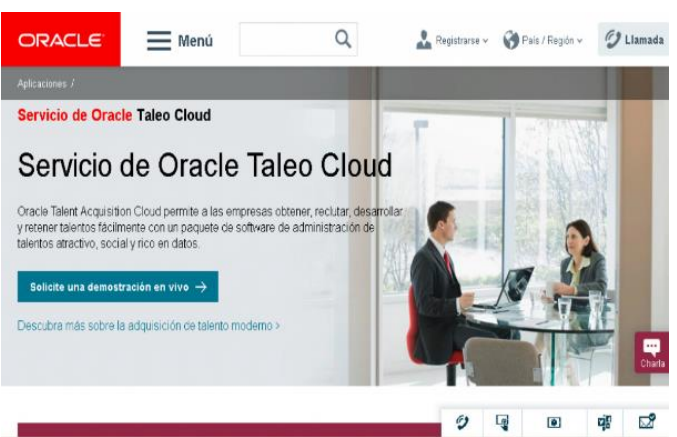

Figura 1. Servicio de Oracle Taleo Cloud (2019).

Tomado de www.comparasoftware.com/oracle-taleo

\section{Novatech}

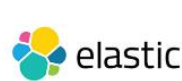

Descubra lo esperado,
Revele lo inesperado. BIG DATA

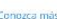

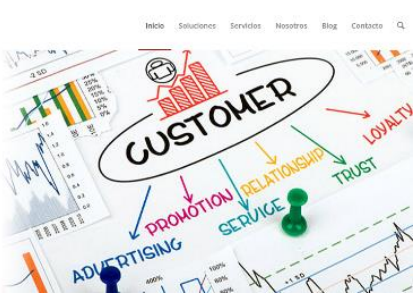

Figura 3. Empresa Novatech (2019).

Tomado www.ingelsi.com.ec/big-data

\section{Empresas que reclutan vía Big Data}
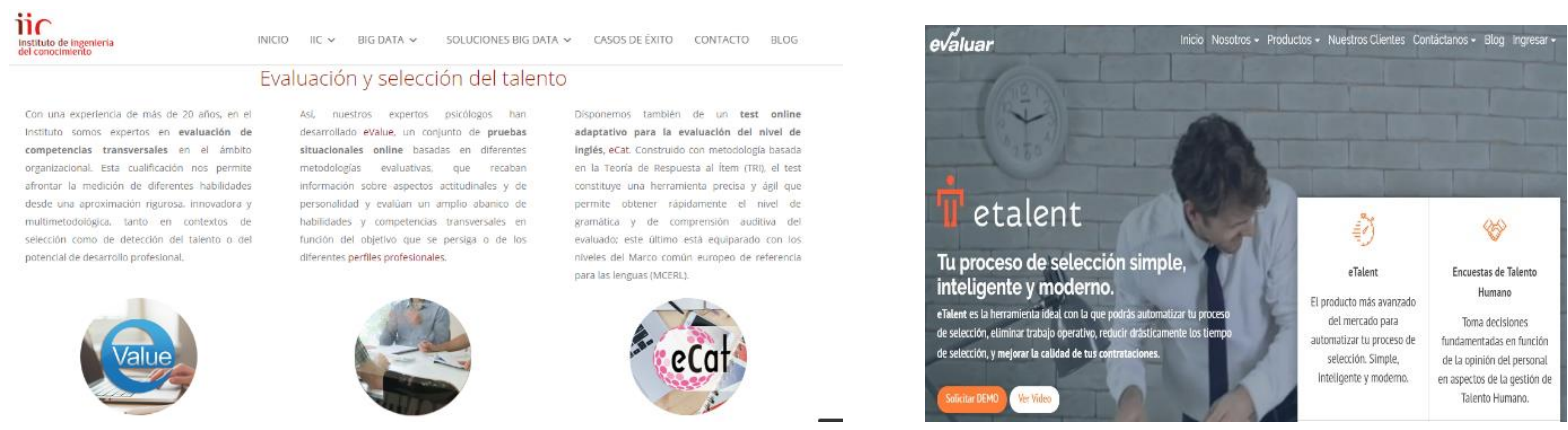

Figura 4. Instituto de ingeniería del conocimiento (2019).

Figura 5. Empresa evaluar (2019).

ww.iic.uam.es/soluciones/recursos-humanos/evalue

Tomado de www.evaluar.com

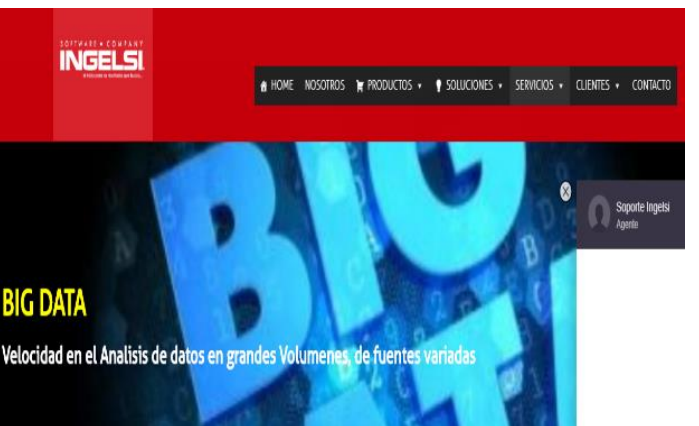

Figura 2. Empresa Ingelsi (2019).

Tomado de www.ingelsi.com.ec/big-data 
ISSN: 2602-8506

Vol. 3, N4., p.116-132, octubre - diciembre, 2019

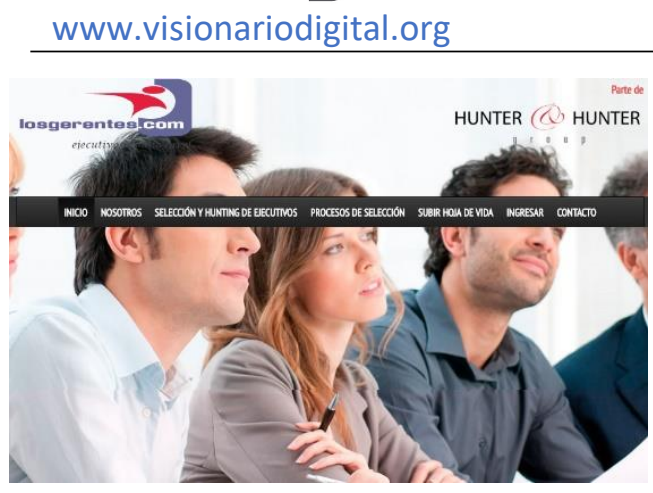

Figura 6. Hunter\&Hunter (2019).

Extraído de hunterandhuntergroup.com

En la actualidad la realidad de los colaboradores en las compañías es cada vez más compleja por diferentes factores: En el mismo entorno laboral se debe establecer la convivencia de diferentes generaciones (baby boomers, generación $\mathrm{X}$, millennials, etc.), convivir también con la tecnología en todos los aspectos de la vida, el trabajo en sí mismo, la atracción y la retención de personal que se ha convertido en una verdadera cacería de talento, establecer estrategias de compensaciones y beneficios, etc. Toda esta vorágine implica la creación de datos y variables que son "medibles” y “analizables” (Quintana, 2019)

$\mathrm{Y}$ vemos que muchas empresas manejan muchos datos siendo y considerando a la gestión de talento Humano como el puntal principal en las empresas por los datos que se manejan y entre estos están:

- Datos de los colaboradores - datos típicos de legajos o carpetas de personal.

- Datos de nómina, remuneraciones y beneficios.

- Datos de registro de entradas y salidas

- Datos de desempeño y potencial.

- Datos de capacitaciones.

- Datos de competencias, planes de carrera y desarrollo

- Datos de Aportes del IESS

- Datos de administración del tiempo (Quintana, 2019) 
ISSN: 2602-8506

Vol. 3, N4., p.116-132, octubre - diciembre, 2019

La información bien administrada nos brinda un plus en el conocimiento, y ya sabemos lo que el conocimiento bien administrado puede generar, aparte de la misma función que tenga cada una de nuestras organizaciones. El poder reunir toda esa información que tenemos dando vueltas sobre nuestros colaboradores e interpretarla de la manera correcta, nos va a dar un entendimiento claro y una visión holística de cada uno de ellos. (UBJ, 2019)

Entonces, entran en juego las soluciones de Big Data y Analíticos: esta se ha convertido en una herramienta necesaria que permite capturar toda esa información, consolidarla, estructurarla y "mostrarla" de una manera que tenga sentido para nosotros y nos ayude a tomar las decisiones correctas.

Hay que recordar que entender lo qué pasa con nuestros colaboradores es entender lo que pasa con el negocio.

\section{Conclusiones}

Además de capturar y mostrarnos los datos, estas herramientas ayudan a predecir qué va a pasar con nuestros colaboradores en los próximos años. Muchos cuando escuchan estas aseveraciones creen que se trata de una película de ciencia ficción al puro estilo de Spielberg, pero esta es la realidad contemporánea que nos obliga a mantenernos actualizados y obviamente debemos reconocer el valor que estas herramientas le dan al área de talento humano y al negocio: poder predecir qué va a pasar con nuestra gente es, de alguna manera, poder predecir qué va a pasar con el negocio.

No es novedad que desde hace un buen tiempo los profesionales de talento humano utilizan herramientas de análisis para asistirlos en la toma de decisiones. Lo que es importante destacar es qué tipo de analíticos utilizamos y para qué pueden servirnos.

\section{Existen 4 tipos de analíticos:}

- Analíticos Descriptivos

- Analíticos Diagnósticos

- Analíticos Predictivos

- Analíticos Prescriptivos

Los primeros dos tipos de analíticos (Descriptivos y Diagnósticos) son los que tradicionalmente utilizamos. Se relacionan con el análisis de información que ya ocurrió o 
que está ocurriendo y sus causas. Algunos ejemplos aplicados a Talento Humano son: índices de rotación de personal, evoluciones salariales, indicadores de desempeño, historiales de formación, etc. Este tipo de analíticos requiere de un análisis para interpretar las variables y tomar las acciones y medidas necesarias.

El segundo grupo de analíticos (Predictivos y Prescriptivos) son la nueva tendencia en Talento Humano, aquí es donde entran en juego los conceptos de "Big Data". Este tipo de analíticos recolectan toda la información de las múltiples fuentes de talento humano, las procesan y determinan mediante estudio de patrones de comportamiento cuáles son los posibles escenarios de desenlaces y qué acciones se pueden realizar para mitigar o cambiar los parámetros descritos. Entonces, tomando información del área hard y soft de Talento Humano, podremos alcanzar una solución que contribuye a determinar y predecir cuál es el futuro de un colaborador.

Accediendo a la información "histórica" de resultados de evaluaciones de desempeño y matriz de potencial desempeño de un colaborador, historial de formación y capacitaciones de interés, evaluando cuáles son las competencias más fuertes, el plan de carrera sugerido para su posición, su evolución salarial, el índice de intereses, este tipo de analíticos pueden indicar cuándo el colaborador va a estar listo para cubrir y acceder a otra posición, predecir cuáles son los puestos donde mejor podría desempeñarse, qué tipo de capacitación necesita, cuál es el riesgo de perder a ese colaborador y cuándo podría ocurrir, entender cuáles son las tareas de mayor desarrollo y sugerirnos dónde vamos a sacar el mayor rédito de sus habilidades.

En resumen, la inteligencia artificial y el concepto de Big Data permite el procesamiento de los datos y la predicción de diferentes escenarios, ahorrando significativamente los tiempos de recolección de datos, elaborando y armando reportes y definir de planes de acción, permitiendo que el área de Talento Humano pueda responder a las exigencias del negocio de manera inmediata, con información fidedigna y estadística.

Talento Humano es el área de mayor estrategia en el negocio manejando de manera acertada el conocimiento permitirá tomar decisiones más acertadas. La Dirección de Talento Humano se ve directamente beneficiada por esto, ya que tiene visibilidad de los datos de los colaboradores y le permite tomar decisiones más atinadas. 
Gran parte este beneficio va a estar determinado por el tipo de analíticos que utilicemos. Si se ocupan los tradicionales, donde observamos la información de lo ya acontecido, el beneficio principal será poder contar con los elementos necesarios para tomar una decisión; en cambio, si utilizamos los analíticos predictivos y el Big Data estaremos siempre dando un paso más allá, porque podremos no sólo entender lo que pasó sino también lo que va a suceder y, podremos automáticamente determinar qué podemos hacer al respecto. El beneficio es incalculable y dependerá del uso que el área quiera darle a esa información.

Big Data lleva a la gestión del talento humano a otro nivel. Con herramientas que logran estructurar toda la información del área, y predecir qué puede llegar a pasar proponiendo alternativas de resolución. Es fundamental entender qué va a ocurrir con nuestros colaboradores y, por ende, con nuestro negocio.

Big Data es la solución que dará a los departamentos de talento humano la trascendencia ya que al manejar la información se transforma en el departamento de mayor valor en el negocio.

\section{Referencias Bibliográficas}

Calle, A. (3 de diciembre de 2018). Focus. Obtenido de https://focushumanconsulting.com/2018/12/03/big-data-que-es-y-como-usarlo-en-rrhh/

Gil González, E (2015). Big Data, privacidad y protección de datos. España: Boletín Oficial del Estado.

Grande, L. S. V. (2004). Gestión por competencias: el reto compartido del crecimiento personal y de la organización. Esic Editorial.

Grupo Castilla. (10 de mayo de 2016). Obtenido de https://www.grupocastilla.es/es/noticias/como-puedo-aprovechar-el-big-data-enrecursos-humanos

Houghton Mifflin Harcourt .Patil D. (2011) Building Data Science Teams. United States of América: O'Reilly

Larrosa, J. (s.f.). Economía del Sofware. Obtenido de https://econsoft.blogspot.com/2015/04/

Mahiques, M. (19 de Julio de 2012). The club of compulsive Readers. Obtenido de https://theclubofcompulsivereaders.blogspot.com/2012/07/que-es-big-data.html

Mayer-Schönberger, V. (2013). Big Data: A Revolution That Will Transform How We Live, Work, and Think.

Mihala, D. (2019). Big Data. Obtenido de https://www.oracle.com/es/applications/humancapital-management/features/bigdata/

Quintana, J. P. (2019). Blog P. Obtenido de https://blog.portinos.com/novedades/tecnologia/big-data-y-analytics-aplicado-almanagement-de-rrhh 
ISSN: 2602-8506

Vol. 3, N4., p.116-132, octubre - diciembre, 2019

Ranstad. (13 de diciembre de 2016). Obtenido de https://www.randstad.es/tendencias360/elbig-data-en-los-recursos-humanos/

Rua, M. (15 de Julio de 2012). La Nación. Obtenido de https://www.lanacion.com.ar/lifestyle/el-nuevo-oro-se-llama-big-data-nid1490304

Rua, M. (s.f.). Zona de Promesas. Obtenido de https://marcosmd.wordpress.com/2012/07/15/el-nuevo-oro-se-llama-big-data/

UBJ. (2019). Obtenido de https://www.ubjonline.mx/rol-del-liderazgo-en-la-gestion-deltalento-humano/

Vallejo, Ma. Cómo ayudan las TIC en el empoderamiento social de los individuos.

Corporación Colombia Digital, 2013.

Vega, J. J. (2015). Conociendo Big Data. Revista Facultad de Ingeniería, 65.

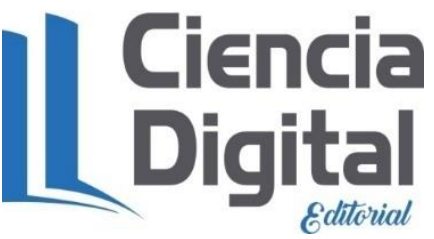




\section{PARA CITAR EL ARTÍCULO INDEXADO}

Santillán Marroquín, W., Paredes García, H., Bermeo Castillo, C., \& Mera Manotoa, H. (2019). Big

Data en la gestión del talento humano. Visionario Digital, 3(4), 116-132.

https://doi.org/10.33262/visionariodigital.v3i4.984

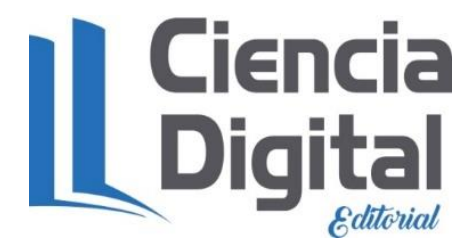

El artículo que se publica es de exclusiva responsabilidad de los autores y no necesariamente reflejan el pensamiento de la Revista Visionario Digital.

El artículo queda en propiedad de la revista y, por tanto, su publicación parcial y/o total en otro medio tiene que ser autorizado por el director de la Revista Visionario Digital.
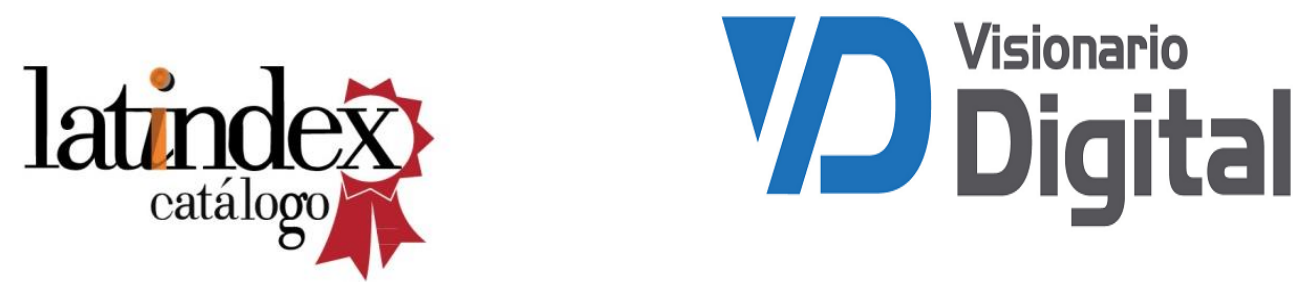